\title{
Cancer Testis Antigens and Immunotherapy: A new Dawn
}

\author{
Bingcan Zhang ${ }^{1,2,3, *}$ \\ ${ }^{1}$ State Key Laboratory of Oncogenes and Related Genes, Ninth People's Hospital, Shanghai Jiao Tong University School of Medicine, \\ Shanghai, China \\ ${ }^{2}$ Shanghai Institute of Precision Medicine, Shanghai, China.Shanghai Institute for Advanced Immunochemical Studies, ShanghaiTech \\ University, Shanghai, China \\ ${ }^{3}$ Key Laboratory of Cell Differentiation and Apoptosis of Chinese Ministry of Education, Shanghai Jiao Tong University School of \\ Medicine, Shanghai, China
}

\begin{abstract}
Immunotherapy for cancer has been recognized as the fourth therapeutic method after surgery, radiotherapy and chemotherapy, which can prevent postoperative metastasis and recurrence and reduce or even eliminate the toxic and side effects of chemoradiotherapy. The development of successful immunotherapy strategies need to use cancer antigens which can be identified by the host's immune system. This method's ability in causing antitumor immune response has been fully proved, but it also faces enormous risks and challenges, as finding the highly efficient and specific tumor markers is very difficult. Cancer-testis antigens(CTA) are a special kind of tumor antigens with normal expression restricted to male germ cells in the testis but not in adult somatic tissues. The immune privileged status of CTA gives tumor specificity and makes it an ideal candidate for targeted immunotherapy biomarkers. Here, we briefly review the research history, expression characteristics of CTA, molecular mechanisms of CT gene, and the bright future of immunotherapy in cancer treatment.
\end{abstract}

\section{Introduction}

The search for a cure for cancer has been lasting for a long time. Cancer remains by far one of the most threatening diseases to humans 1 . Traditional treatments include surgery, radiation and chemotherapy, which can destroy cancer cells but also do harm to normal tissues. These toxic side effects greatly reduce the quality of life of cancer patients. The fourth modality of immunotherapy has been well documented since 1890 when Coley demonstrated that bacterial products (Coley toxins) had benefits for inoperable cancers and the subsequent application of Bacillus Calmette-Guerin (BCG) and other crude immunostimulants showed benefits that led to regulatory approval of their use in some solid tumors such as bladder cancer 2. Tumor immunotherapy is simply to use a variety of means to activate the body's anti-tumor immune response, and finally eliminate and kill tumor cells. Scientific research has shown that tumor cells can trick the body's immune system into mistaking them for the body's own normal tissue cells to avoid being attacked and destroyed by the immune system 3. The goal of tumor immunotherapy is to expose the deception of tumor cells, activate the immune system, recognize, attack and finally kill tumor cells. Although immunotherapy has been shown to be a promising strategy for treating different human cancers, one of the major limitations remains the lack of a tumorassociated antigen (TAA) that can be an effective target 4.

* $\overline{\text { Corresponding author: sophia0909@sjtu.edu.cn }}$
Cancer-testis antigens(CTA) are a category of tumor antigens with normal expression restricted to male germ cells in the testis. In some cases, CTA are also expressed in ovary and in trophoblast. They are important cell surface proteins during reproductive development. In malignancy, this gene regulation is disrupted, resulting in CTA expression in a proportion of tumors of various types 5. However, their expression in different tumors is heterogeneous and related to the degree of tumor progression. They are expressed wildly and meaningfully in melanoma, liver cancer, bladder cancer and pediatric tumors such as neuroblastoma, etc. So they are ideal biomedical marker to be used in T lymphocytic or antigen-specific and targeted cellular immunotherapy.

CTA can be classified as CT-X antigens and non-X CT antigens based on whether their genes are encoded by the $\mathrm{X}$ chromosome. CT-X antigens are highly expressed in spermatogonial cells and tend to express multiple CT antigens simultaneously, while non-X CT antigens are distributed throughout the genome and are usually expressed in sperm cells 6 . Up to $10 \%$ of the genes on the $\mathrm{X}$ chromosome were identified as belonging to the CT-X family[5].

According to the current information in the CTdatabase (http://www.cta.lncc.br) and GeneBank (https://www.ncbi.nlm.nih.gov/gene), there are now more than 200 gene families, classified into 51 gene families 9 , that are strongly upregulated in cancer where the normal expression is highly biased to the germline. Not all of these have been shown to be capable of 
eliciting immune responses but are collectively termed the $\mathrm{CT}$ antigens.

\section{History: the past and the present}

In 1991, Thierry Boon and his colleagues successfully cloned the first tumor antigen in a ground breaking study, which significantly changed the field of tumor immunology 10. MAGE-A genes elicit an HLA class Idependent cytotoxic response in sensitised lymphocytes against the melanoma cell line MZ2-MEL, and because of that they were discoverd firstly 11 . One of the most special characteristics of the antigen in question, MAGE$\mathrm{A} 1$, is that it is normally expressed in the germline but not in other normal tissues. But it was not immediately recognized at that time. Then according to subsequent analysis of the pattern of normal tissue expression, all of the cancer antigens initially cloned using cytotoxic Tcell clones and autologous tumor mRNA were found to have testis restricted expression among normal adult tissues 12. The MAGE-A family is comprised of 12 highly homologous genes clusted at Xq28. The genes are characterized by the presence of a conserved domain (MHD, MAGE Homology Domain). MAGE-A gene expression occurs in the normal human germline, and in a wide range of tumors. MAGE-A1 is expressed in a wide variety of human cancers at the RNA and protein level. Antigen-specific immune responses can be induced by immunization with MAGE-A1 .

Based on these findings, other tumor antigens encoded by testicular restriction genes, including BAGE and GAGE, were also found using cytotoxic $\mathrm{T}$ cells isolated from the same patient who discovered MAGEA1 14. In the same way, SYCP1 antigens were also isolated.

The genetic methods of cloning MAGE, BAGE and GAGE were technically challenging because they required the establishment of autologous CTL lines and tumor cell lines from the same patient, which was not easy to achieve for most epithelial tumor types 16 . To overcome these limitations, a technique called SEREX was designed to isolate tumor antigens that elicit high titer immunoglobulin $\mathrm{G}$ responses in human hosts, known as SEREX for recombinant tumor cDNA expression libraries 17 .

Then these initial discoveries were soon extended through the identification of antigens recognized by antibodies in the sera of cancer patients. One of the first antigens discovered by this approach was NY-ESO-1 that appears to be the most immunogenic of the CT antigens and has become the major focus of experimental therapeutic vaccine development within the Ludwig Institute 18. NY-ESO-1 antibodies are present in the serum of a significant number of cancer patients. Up to $50 \%$ of patients bearing NY-ESO-1-expressing tumors spontaneously develop specific antibody responses during the course of their disease. Serum antibody frequencies to NY-ESO-1 appear related to gene expression in the tumor, and may increase with higher stage or grade. However, it is reported in one of the recent researches that NY-ESO-1 induces strong immune responses in cancer patients but has limited objective clinical responses to NY-ESO-1 expressing tumors due to effect of competitive negative signaling from immune-checkpoints and immune-suppressive tumor microenvironment. The major aim of researchers now is to counter potential roadblocks in therapeutic pathways encompassing NY-ESO-1. This can potentially be achieved via combination therapy to increase the efficacy of NY-ESO-1 specific immunotherapeutic interventions 19.

SEREX clones of recombinant human melanoma cDNA from human melanoma using autologous serum were serologically analyzed and several neomelanoma antigens with immunogenicity in autologous hosts were identified [20]. A variety of antigens have been detected in subsequent work, such as CT7/ MAGE-C1, SCP-1, HOM-TES-85, cTAGE-1, OY-TES-1, CAGE and so on.

Emerging data suggest that they may play a key role in many important cellular processes, such as signal transduction, transcriptional regulation, presumed protooncogenes and cell growth, but there are few research achievements on the function of CT antigens, and people only have a relatively clear understanding of the function of several CT antigens. For example, CAGE-1 is predominantly expressed during postmeiotic stages and CAGE-1 is a novel component of the acrosome of mammalian spermatids and spermatozoa 21 . SCP-1 is an important component of the synaptic complex and is closely related to the pairing, exchange and separation of chromosomes 22. SPANXN2 regulates TGCT cell migration via EMT- and AKT-related proteins although its role in the occurrence and development of TGCT remains to be fully elucidated 23 .

In addition to immunogenicity, CTAs have oncogenic functions including support of tumor growth (i.e., SSX and CAGE), evasion of apoptosis (i.e., MAGE $\mathrm{A} / \mathrm{B} / \mathrm{C}, \mathrm{GAGE}$ and PAGE4), and induction of invasion and metastasis (i.e., MAGE C2, CAGE, GAGE, XAGE and CT45A1) 24.

\section{Expression: heterogeneity among different tumors}

The expression of CT-X antigens vary greatly among different types of tumors. For example, CT-X antigens are frequently expressed in bladder, lung, ovarian and hepatocellular carcinomas, and melanoma, according to reverse transcription polymerase chain reaction (RTPCR) analysis, but are rarely observed in the kidney or colon, gastric cancer, leukemia and lymphoma cells.

Specific CTA is expressed at different frequencies in different types and subtypes of tumors. Melanoma was most frequently expressed, followed by sarcoma, lung cancer, and breast cancer. In general, the expression of antigens in tumor tissues has the following three characteristics.

Firstly, the same CT antigen is expressed differently in different tumor tissues. For example, NY-TLU-57, GAGE1, SAGE1 were expressed more frequently in tumor samples than in healthy tissues 25 . But SAGE1 were only expressed in 3-5\% of ER-negative and $0-2 \%$ 
of ER-positive cancers 26. Surprisingly, it is detected that no SAGE1 is expressed in the 67 lung cancers (mainly non-small lung cancer), while MAGE-A proteins were present in $15 \%$ and $7-16 \%$ of these tumor types, respectively 27.

Secondly, the expression frequency of different CT antigens in the same tumor tissue is also different. In a former research, scientists analysed the expression pattern of OCT2, SSX2-4, and SAGE1 in 36 SS cases and four intratubular SS (ISS) as well as a series of normal testis samples throughout development and it is concluded that OCT2, SSX and SAGE1 reveal the phenotypic heterogeneity of spermatocytic seminoma reflecting distinct subpopulations of spermatogonia 28 .

Thirdly, CTA expression tends to be aggregated, that is, it is found that some tumor specimens express multiple CT antigens simultaneously, while others will be completely free of CT antigens. The highest frequency of expression was found in esophageal squamous cell carcinomas: Positive staining for MAGEA, CT45, CT7, SAGE1, GAGE, NXF2, NYESO-1, and CT10 was observed in $57 \%, 38 \%, 33 \%$, $33 \%, 29 \%, 29 \%, 19 \%$, and $14 \%$ of squamous cell carcinomas, respectively, suggesting a potential diagnostic role for cancer-testis antigens in the evaluation of premalignant squamous lesions 29.

\subsection{Melanoma}

Melanoma is the first malignant tumor with testicular antigen (CTAs) expression and immunogenicity documented. Some cancer testis antigens with strong metastatic potential have been shown to be expressed in melanoma samples 30. A kind of CTA called preexpressivelyexpressed melanoma antigen (PRAME), is expressed in many primary and metastatic melanomas and is co-expressed with CLASS HLA I in many of these melanomas. In addition, PRAMe-specific T cells have been shown to recognize PRAMe-positive melanoma cell lines, making them targets for immunotherapy 31. Some CTAs' expression has been shown to correlate with disease progression or clinical outcomes. For example, expression of MAGE-A1, MAGE-A4 and NY-ESO-1 was negatively associated with recursion-free survival in a large number of melanoma patients 32 .

\subsection{Esophageal cancer}

In esophageal cancer, although the relationship between the expression and prognosis of MAGE-A, NY-ESO-1, TTK and LAGE-1 remains controversial, the MAGE-A, NY-ESO-1, LAGE-1 and TTK are highly expressed, which can induce specific CTL cells for tumor cells to produce special effect. Some clinical trials have shown that immune treatment in patients with esophageal cancer is safe and effective, and provides a new treatment strategy for the treatment of esophageal cancer 33.

\subsection{Non-small cell lung carcinoma}

The MAGE-A3 cancer/testis antigen is frequently expressed in non-small cell lung cancer (NSCLC) and vaccination with MAGE-A3 in patients with MAGE-A3positive NSCLC has shown promising results. One of results shows that the $\mathrm{CT}$ antigens GAGE, NY-ESO-1 and SP17 are expressed in a considerable proportion of NSCLC and may therefore serve as candidate targets for immunotherapeutic treatments of this disease. Furthermore, GAGE and NY-ESO-1 were present in more than $50 \%$ of the tumor cells in $63.6 \%(28 / 44)$ and $70 \%(14 / 20)$ of the positive cases, respectively 34 .

\subsection{Ovarian cancer}

Based on multiple independent large databases, including Genotype-Tissue Expression (GTEx), and human proteomic and TCGA data, one group recently systematically explored the molecular landscape of CT genes in 19 cancer types. CTA family members in ovarian cancer reported to date include MAGE genes, NY-ESO-1, SSX, and CT45, which are categorized as CT-X antigens, and BORIS, PRAME, PIWIL, and AKAP3/4, which are categorized as non-X CTAs 35.

\subsection{Adenoid cystic carcinoma of the head and neck}

Adenoid cystic carcinoma of the head and neck is a rare but highly malignant tumor. According to a former research, eighty-four cases of ACC were identified, and Expression of NY-ESO-1 was found in 48/84 (57.1\%) and of pan-MAGE in 28/84 (31.2\%) 36. As such a significant fraction of ACC patients show expression of the cancer testis antigens NY-ESO-1 and pan-MAGE, subgroup of patients with a poor prognosis carries antigens targetable by specific immunotherapy 37 .

\subsection{Breast cancer}

Breast cancer is still the most common cancer affecting women in the whole world, posing a serious threat to women's lives and health. It has been indicated that CT antigens expression is closely related to the ER status 38 . Other CTAs such as HORMAD1, CXorf61, ACTL8 and PRAME were found to be highly expressed in the breast cancers that have basal cell properties 39. Furthermore, there are many other CTAs that predict invasion and metastasis of breast cancer such as ATAD2 and GPAT2 40.

There are also personalized CTA expressions in other tumors, which will not be repeated here.

\section{Molecular mechanisms of CT gene expression}

CT genes share a common mechanism of regulation at the transcriptional level 42. A lot of researches have indicated that epigenetic regulation is a key mechanism in the transcriptional regulation of CT genes 43 . The 
mechanism of CTA activation includes the following several categories.

\subsection{DNA hypomethylation in CT gene activation}

DNA methylation plays an important role in the epigenetic hierarchy of CT gene expression. DNA hypomethylation harmonizes $\mathrm{CT}$ gene promoters in EOC and is associated with advanced disease.

Initial evidence suggests that activation of MAGEA1 in cancer cells is caused by hypomethylation of promoter DNA 44. It is reported that in ovarian cancer, DNA hypomethylation drives expression of BORIS, which is partly determined by the global DNA methylation status 45 . And the same was subsequently found in NY-ESO-1, MAGE genes and some other CT genes.

\subsection{Histone modification and modulation}

Histone deacetylation and DNA methylation play a central role in the control of tumor gene expression, including transcriptional inhibition of tumor suppressor genes and genes involved in chemotherapy sensitivity 46 . In a former research, it is reported that repression of NYESO-1, MAGE-A1, and MAGE-A3 coincided with DNA hypermethylation, recruitment and binding of polycomb group proteins, and histone heterochromatin modifications within the promoters of these genes 47 . However, up to now, studies on the effect of histone modification on CT gene expression are limited, and most of the current studies usually adopt pharmacological methods.

\section{Roles in cancer immunotherapy}

CT antigens can be combined with exosomes for antigenic presentation. Several CT antigens are expressed in exosomes and corresponding tumors. For example, MAGE-B4 gene expression in urinary exosomes of bladder cancer patients was higher than normal control samples 48. And testicular specific lactate dehydrogenase $\mathrm{C} 4$ was up-regulated in serum exosomes of breast cancer patients 49.Because immunogenic $\mathrm{CT}$ antigens are selectively expressed in tumor tissues and exosomes, they have the potential to be used as antigen-specific cancer vaccines or diagnostic markers 50 .

CTAs are promising targets for cancer immunotherapy due to their expression in cancers and their rarity in normal tissues. With the identification of CTA peptides and clinical trials of CTA multipeptide vaccines, establishing personalized CTA peptide vaccines has become possible. Challenges remaining include the search for promising targets, identification of additional immunogenic CTA peptides, choice of suitable clinical settings, and development of feasible combination therapy 51.Humoral immune responses and cellular immune responses against NY-ESO-1 and MAGE-A3 have been detected 52, and the restricted epitopes have been identified as the recognition sites for CD8+ cytotoxic T lymphocytes (CTLs) 53.

To date, five tumor immunotherapy pathways based on CT genes/antigens have been identified:

A. Antigen-presenting cell approach: use CT antigen peptide to incubate autoantigen presenting cells, and then make vaccine into tumor cells expressing CT proteins and corresponding HLA-i molecules to induce specific CTL response, which can be enhanced by IL-2;

B. Melanoma cell inoculation path, namely, melanoma cells expressing CT protein are made into vaccine for tumor patients;

C. Gene transfection pathway, using the virus as the vector, carrying THE CT gene to make a vaccine, to the tumor patients inoculation;

D. Dendritic cell inoculation approach, that is, using CT antigen peptide to incubate monocyte derived dendritic cells, and then inoculated into the tumor patients;

E. The method of antigen peptide inoculation is CT antigen peptide directly inoculated into the body of tumor patients, mainly MAGE and NY-ESO-1.

In summary, CTA is an excellent target for anticancer drug discovery, tumor therapy and diagnostic biomarkers, and is also valuable in immunotherapy, tumor genesis and malignant progression research.

\section{Conclusion}

Cancer testis antigens are normal testicular antigens that are abnormally expressed in tumor cells. Because CT antigens are widely expressed in a variety of tumors, they are ideal molecules for immunotargeting and for the development of cancer vaccines for use in $\mathrm{T}$ lymphocytic or antigen-specific and targeted cellular immunotherapy. Many outstanding scientists have made great contributions to the field of CTA, and the discoveries of this field have opened up new ideas and enticements for cancer treatment and a bright future.

However, the researches in this field are still not thorough enough. At present, we do not have a clear understanding of the role of CT genes in human normal tissues and the expression characteristics of different CTA in different tumors. Current research focuses on the development and study of CTA functional cell pathways, the establishment of different tumor specific CTA expression and other aspects. In addition, in the actual experiment, a reliable evaluation mechanism must be established to determine whether a CTA gene plays a role in the upstream. The method of cell inoculation can be adopted to determine the true role of the gene through the survival performance of the cell.

Despite the promise of using CTA-driven strategies to treat cancer patients, the complexity of human malignancies requires new standards to be established for all of the scientists working in the field to verify the practical applicability of CTAS. 


\section{References}

1. Siegel R, Ma J, Zou Z, Jemal A. Cancer statistics, 2014. CA Cancer J Clin 2014;64(1):9-29. PubMed PMID:24399786.

2. Kirkwood JM, Butterfield LH, Tarhini AA, Zarour H, Kalinski P, Ferrone S. Immunotherapy of cancer in 2012. CA Cancer J Clin. 2012;62(5):309-335. doi:10.3322/caac. 20132

3. Gajewski TF, Schreiber H, Fu YX. Innate and adaptive immune cells in the tumor microenvironment. Nat Immunol. 2013;14(10):1014-1022. doi:10.1038/ni.2703

4. Fox BA, Schendel DJ, Butterfield LH, et al. Defining the critical hurdles in cancer immunotherapy. J Transl Med 2011;9(1):214. PubMed PMID:22168571.

5. Scanlan MJ, Gure AO, Jungbluth AA, Old LJ, Chen YT. Cancer/testis antigens: an expanding family of targets for cancer immunotherapy. Immunol Rev. 2002;188:22-32. doi:10.1034/j.1600065x.2002.18803.x

6. Q. Zhao, O.L. Caballero, A.J.G. Simpson, R.L. Strausberg Differential evolution of MAGE genes based on expression pattern and selection pressure PLoS One, 7 (2012), p. e48240

7. B.J. Stevenson, C. Iseli, S. Panji, M. Zahn-Zabal, W. Hide, L.J. Old, et al. Rapid evolution of cancer/testis genes on the $\mathrm{X}$ chromosome BMC Genomics, 8 (2007), p. 129

8. Ross MT, Grafham DV, Coffey AJ, et al. The DNA sequence of the human $\mathrm{X}$ chromosome. Nature. 2005;434(7031):325-337. doi:10.1038/nature03440

9. L.G. Almeida, N.J. Sakabe, A.R. deOliveira, M.C.C. Silva, A.S. Mundstein, T. Cohen, et al. CTdatabase: a knowledge-base of high-throughput and curated data on cancer-testis antigens Nucleic Acids Res., 37 (2009), pp. D816-D819

10. van der Bruggen, P., Traversari, C., Chomez, P., Lurquin, C., De Plaen, E., Van den Eynde, B., Knuth, A., and Boon, T. (1991) A gene encoding an antigen recognized by cytolytic $\mathrm{T}$ lymphocytes on a human melanoma. Science (New York, N.Y 254, 1643-1647 PMID 1840703

11. Anil Suri (2006) Cancer testis antigens - their importance in immunotherapy and in the early detection of cancer, Expert Opinion on Biological Therapy, 6:4, 379-389, DOI: 10.1517/14712598.6.4.379

12. Chen, Y. T., Stockert, E., Chen, Y., Garin-Chesa, P., Rettig, W. J., van der Bruggen, P., Boon, T., and Old, L. J. (1994) Identification of the MAGE-1 gene product by monoclonal and polyclonal antibodies. Proceedings of the National Academy of Sciences of the United States of America 91, 1004-1008 PMID 8302824

13. De Smet, C., Lurquin, C., van der Bruggen, P., De Plaen, E., Brasseur, F., and Boon, T. (1994) Sequence and expression pattern of the human
MAGE2 gene. Immunogenetics 39, 121-129 PMID 8276455

14. Boël P, Wildmann C, Sensi ML, et al. BAGE: a new gene encoding an antigen recognized on human melanomas by cytolytic $\mathrm{T}$ lymphocytes. Immunity. 1995;2(2):167-175. doi:10.1016/s10747613(95)80053-0

15. De Backer $\mathrm{O}$, Arden $\mathrm{KC}$, Boretti $\mathrm{M}$, et al. Characterization of the GAGE genes that are expressed in various human cancers and in normal testis. Cancer Res. 1999;59(13):3157-3165.

16. Chen YT, Gure AO, Tsang S, et al. Identification of multiple cancer/testis antigens by allogeneic antibody screening of a melanoma cell line library. Proc Natl Acad Sci USA 1998;95(12):6919-6923. PubMed PMID:9618514.

17. Li G, Miles A, Line A, Rees RC. Identification of tumour antigens by serological analysis of cDNA expression cloning. Cancer Immunol Immunother 2004;53(3):139-143. PubMed PMID:14722670.

18. Chen, Y. T., Scanlan, M. J., Sahin, U., Tureci, O., Gure, A. O., Tsang, S., Williamson, B., Stockert, E., Pfreundschuh, M., and Old, L. J. (1997) A testicular antigen aberrantly expressed in human cancers detected by autologous antibody screening. Proceedings of the National Academy of Sciences of the United States of America 94, 1914-1918 PMID 9050879

19. Raza A, Merhi M, Inchakalody VP, et al. Unleashing the immune response to NY-ESO-1 cancer testis antigen as a potential target for cancer immunotherapy. J Transl Med. 2020;18(1):140. Published 2020 Mar 27. doi:10.1186/s12967-02002306-y

20. Türeci O, Sahin U, Schobert I, et al. The SSX-2 gene, which is involved in the $\mathrm{t}(\mathrm{X} ; 18)$ translocation of synovial sarcomas, codes for the human tumor antigen HOM-MEL-40. Cancer Res. 1996;56(20):4766-4772.

21. Alsheimer M, Drewes T, Schütz W, Benavente R. The cancer/testis antigen CAGE-1 is a component of the acrosome of spermatids and spermatozoa. Eur $\mathrm{J}$ Cell Biol. 2005;84(2-3):445-452. doi:10.1016/j.ejcb.2004.11.003

22. Wobus M, List C, Dittrich T, et al. Breast carcinoma cells modulate the chemoattractive activity of human bone marrow-derived mesenchymal stromal cells by interfering with CXCL12. Int J Cancer. 2015;136(1):44-54. doi:10.1002/ijc. 28960

23. Zhu F, Bo H, Liu G, Li R, Liu Z, Fan L. SPANXN2 functions a cell migration inhibitor in testicular germ cell tumor cells. PeerJ. 2020;8:e9358. Published 2020 Jun 23. doi:10.7717/peerj.9358

24. Mahmoud AM. Cancer testis antigens as immunogenic and oncogenic targets in breast cancer. Immunotherapy. 2018;10(9):769-778. 
25. Atanackovic D, Blum I, Cao Y, et al. Expression of cancer-testis antigens as possible targets for antigenspecific immunotherapy in head and neck squamous cell carcinoma. Cancer Biol Ther. 2006;5(9):12181225. doi:10.4161/cbt.5.9.3174

26. Chen YT, Ross DS, Chiu R, et al. Multiple cancer/testis antigens are preferentially expressed in hormone-receptor negative and high-grade breast cancers. PLoS One. 2011;6(3):e17876. Published 2011 Mar 18. doi:10.1371/journal.pone.0017876

27. Maheswaran E, Pedersen CB, Ditzel HJ, Gjerstorff MF. Lack of ADAM2, CALR3 and SAGE1 Cancer/Testis Antigen Expression in Lung and Breast Cancer. PLoS One. 2015;10(8):e0134967. Published $2015 \quad$ Aug 7. doi:10.1371/journal.pone.0134967

28. Lim J, Goriely A, Turner GD, et al. OCT2, SSX and SAGE1 reveal the phenotypic heterogeneity of spermatocytic seminoma reflecting distinct subpopulations of spermatogonia. J Pathol. 2011;224(4):473-483. doi:10.1002/path.2919

29. Chen YT, Panarelli NC, Piotti KC, Yantiss RK. Cancer-testis antigen expression in digestive tract carcinomas: frequent expression in esophageal squamous cell carcinoma and its precursor lesions. Cancer Immunol Res. 2014;2(5):480-486. doi:10.1158/2326-6066.CIR-13-0124

30. Faramarzi S, Ghafouri-Fard S. Melanoma: a prototype of cancer-testis antigen-expressing malignancies. Immunotherapy. 2017;9(13):11031113. doi:10.2217/imt-2017-0091

31. Gezgin G, Luk SJ, Cao J et al. PRAME as a potential target for immunotherapy in metastatic uveal melanoma. JAMA Ophthalmol. 135(6), 541549 (2017).

32. Svobodová S, Browning J, Macgregor D et al. Cancer-testis antigen expression in primary cutaneous melanoma has independent prognostic value comparable to that of Breslow thickness, ulceration and mitotic rate. Eur. J. Cancer 47(3), 460-469 (2011).

33. Zhang Y, Zhang Y, Zhang L. Expression of cancertestis antigens in esophageal cancer and their progress in immunotherapy. J Cancer Res Clin Oncol. 2019;145(2):281-291. doi:10.1007/s00432019-02840-3

34. Gjerstorff MF, Pøhl M, Olsen KE, Ditzel HJ. Analysis of GAGE, NY-ESO-1 and SP17 cancer/testis antigen expression in early stage nonsmall cell lung carcinoma. BMC Cancer. 2013;13:466. Published 2013 Oct 8. doi:10.1186/1471-2407-13-466

35. Xie $\mathrm{K}, \mathrm{Fu} \mathrm{C}$, Wang $\mathrm{S}$, et al. Cancer-testis antigens in ovarian cancer: implication for biomarkers and therapeutic targets. J Ovarian Res. 2019;12(1):1. Published 2019 Jan 4. doi:10.1186/s13048-0180475-z

36. Veit JA, Heine D, Thierauf J, et al. Expression and clinical significance of MAGE and NY-ESO-1 cancer-testis antigens in adenoid cystic carcinoma of the head and neck. Head Neck. 2016;38(7):10081016. doi:10.1002/hed.24403

37. Laban S, Atanackovic D, Luetkens $\mathrm{T}$, et al. Simultaneous cytoplasmic and nuclear protein expression of melanoma antigen-A family and NYESO-1 cancer-testis antigens represents an independent marker for poor survival in head and neck cancer. Int J Cancer. 2014;135(5):1142-1152. doi:10.1002/ijc.28752

38. Li Y, Li J, Wang Y, et al. Roles of cancer/testis antigens (CTAs) in breast cancer. Cancer Lett. 2017;399:64-73. doi:10.1016/j.canlet.2017.02.031

39. Yao J, Caballero OL, Yung WK, et al. Tumor subtype-specific cancer-testis antigens as potential biomarkers and immunotherapeutic targets for cancers. Cancer Immunol Res. 2014;2(4):371-379. doi:10.1158/2326-6066.CIR-13-0088

40. Caron C, Lestrat C, Marsal S, et al. Functional characterization of ATAD2 as a new cancer/testis factor and a predictor of poor prognosis in breast and lung cancers. Oncogene. 2010;29(37):51715181. doi:10.1038/onc.2010.259

41. Pellon-Maison M, Montanaro MA, Lacunza E, et al. Glycerol-3-phosphate acyltranferase- 2 behaves as a cancer testis gene and promotes growth and tumorigenicity of the breast cancer MDA-MB-231 cell line. PLoS One. 2014;9(6):e100896. Published 2014 Jun 26. doi:10.1371/journal.pone.0100896

42. De Smet C, Lurquin C, Lethe B, Martelange V, Boon T. DNA methylation is the primary silencing mechanism for a set of germ line- and tumorspecific genes with a CpG-rich promoter. Mol Cell Biol. 1999;19(11):7327-7335. doi: 10.1128/MCB.19.11.7327.

43. Karpf AR. A potential role for epigenetic modulatory drugs in the enhancement of cancer/germ-line antigen vaccine efficacy. Epigenetics. 2006;1(3):116-120. doi: 10.4161/epi.1.3.2988.

44. De Smet C, De Backer O, Faraoni I, Lurquin C, Brasseur F, Boon T. The activation of human gene MAGE-1 in tumor cells is correlated with genomewide demethylation. Proc Natl Acad Sci U S A. 1996;93(14):7149-7153. doi:10.1073/pnas.93.14.7149

45. Woloszynska-Read A, James SR, Link PA, Yu J, Odunsi K, Karpf AR. DNA methylation-dependent regulation of BORIS/CTCFL expression in ovarian cancer. Cancer Immun. 2007;7:21. Published 2007 Dec 21.

46. Steele N, Finn P, Brown R, Plumb JA. Combined inhibition of DNA methylation and histone acetylation enhances gene re-expression and drug sensitivity in vivo. Br J Cancer. 2009;100(5):758763. doi:10.1038/sj.bjc.6604932

47. Rao M, Chinnasamy N, Hong JA, et al. Inhibition of histone lysine methylation enhances cancer-testis antigen expression in lung cancer cells: implications 
for adoptive immunotherapy of cancer. Cancer Res. 2011;71(12):4192-4204. doi:10.1158/00085472.CAN-10-2442

48. Yazarlou F, Mowla SJ, Oskooei VK, Motevaseli E, Tooli LF, Afsharpad M, Nekoohesh L, Sanikhani NS, Ghafouri-Fard S, Modarressi MH Cancer Manag Res. 2018; 10():5373-5381.

49. Cui Z, Chen Y, Hu M, Lin Y, Zhang S, Kong L, Chen Y Clin Chim Acta. 2020 Apr; 503():203-209.

50. Kothandan VK, Kothandan S, Kim DH, et al. Crosstalk between Stress Granules, Exosomes, Tumour Antigens, and Immune Cells: Significance for Cancer Immunity. Vaccines (Basel). 2020;8(2):172. Published 2020 Apr 8. doi:10.3390/vaccines8020172

51. Wei X, Chen F, Xin K, et al. Cancer-Testis Antigen Peptide Vaccine for Cancer Immunotherapy: Progress and Prospects. Transl Oncol.
2019;12(5):733-

738.doi:10.1016/j.tranon.2019.02.008

52. Thomas R, Al-Khadairi G, Roelands J, Hendrickx W, Dermime S, Bedognetti D, Decock J. NY-ESO-1 based immunotherapy of cancer: current perspectives. Front Immunol. 2018;9:947.

53. Yamaguchi H, Tanaka F, Ohta M, Inoue H, Mori M. Identification of HLA-A24-restricted CTL epitope from cancer-testis antigen, NY-ESO-1, and induction of a specific antitumor immune response. Clin Cancer Res. 2004;10:890-896.

54. Eikawa S, Kakimi K, Isobe M, Kuzushima K, Luescher I, Ohue Y, Ikeuchi K, Uenaka A, Nishikawa H, Udono H. Induction of CD8 T-cell responses restricted to multiple HLA class I alleles in a cancer patient by immunization with a 20 -mer NY-ESO-1f (NY-ESO-1 91-110) peptide. Int J Cancer. 2013;132:345-354. 